\title{
Coronary Artery Calcium Progression Is Associated with Cardiovascular Events Among Asymptomatic Individuals: From the North Texas Primary Care Practice-based Research Network (NorTex-PBRN)
}

\author{
Roberto Cardarelli, DO, MHA, MPH, Alexandria Hall, BA, and Wade Rankin, DO
}

Background: Although incidental coronary artery calcium (CAC) has been established as a surrogate measure for atherosclerotic plaque burden, little is known about its progression and the associated risks. This study looks at the association of select cardiovascular risk factors with the progression of CAC over a 2-year period and the relationship between CAC progression and experiencing a composite cardiovascular disease (CVD) event.

Methods: Repeated CAC measurements were obtained for 311 asymptomatic participants aged $>44$ years, who were recruited from a collaborative network of primary care clinics.

Results: An average of 24.4 months separated scans and CAC scores increased by a mean of 24.45 Agatston units. A total of 113 participants (30\%) demonstrated CAC progression, whereas the rest showed no change or a decrease in CAC over 2 years. In adjusted regression models that controlled for age and sex, the following were associated with 2 -year CAC progression: dyslipidemia, systolic blood pressure, fasting glucose, and non-high-density lipoprotein. Moreover, those with progressive CAC measures were $>4$ times more likely to experience a composite CVD event in 2 years, after controlling for known risk factors.

Conclusions: Overall, several baseline risk factors remained significant after adjusting for age and sex. CAC progression was independently associated with a composite CVD event. (J Am Board Fam Med 2017;30:592-600.)

Keywords: Atherosclerotic Plaque, Blood Pressure, Cardiovascular Diseases, Dyslipidemia, Practice-based Research, Primary Health Care, Risk Factors

Coronary artery calcium (CAC) reflects atherosclerotic plaque burden and constitutes a validated biomarker for the presence and extent of disease. ${ }^{1}$ Studying the significance of CAC in asymptomatic individuals is warranted because the majority of initial coronary heart disease events are acute myo-

This article was externally peer reviewed.

Submitted 7 February 2017; revised 24 May 2017; accepted 26 May 2017.

From the Department of Family \& Community Medicine (RC, WR), University of Kentucky College of Medicine (AH), Lexington.

Funding: This project was supported by the National Institute on Minority Health and Health Disparities (grant P20MD001633).

Disclaimer: The content is solely the responsibility of the authors and does not necessarily represent the official views of the National Institute on Minority Health and Health Disparities or the National Institutes of Health. cardial infarctions or sudden cardiac death. ${ }^{2}$ While CAC scores cannot nullify the prognostic value of standard risk factors, they have been demonstrated to enhance risk stratification based on pretest coronary heart disease risk estimates such as the Framingham index and pooled cohort risk equations. ${ }^{3-6}$ Furthermore, several studies contend that coronary calcification adds prognostic information above and beyond knowledge of traditional risk factors, and that it is the strongest predictor of cardiac events. $^{2,7,8}$ Thus CAC progression may detect changes in premorbid atherosclerosis and identify subjects who would benefit from more aggres-

Conflict of interest: none declared.

Corresponding author: Roberto Cardarelli, DO, MHA, MPH, University of Kentucky, 2195 Harrodsburg Road, Lexington, KY 40504 (E-mail: roberto.cardarelli@uky.edu). 
sive risk factor management and further diagnostic workup. ${ }^{9,10}$

Understanding the relationship between specific risk factors and CAC progression may improve the mechanistic understanding of coronary heart disease. Currently, evidence shows several traditional risk factors for coronary calcification incidence; among them, age and sex are the most prominent. ${ }^{11}$ Less evidence is available for factors related specifically to CAC progression, and less still is consistent. According to Kronmal et al, ${ }^{12}$ certain factors (low-density lipoprotein [LDL] and high-density lipoprotein [HDL]cholesterol) seem to be related only to the risk of incident CAC, not to the progression of existing calcification. Yoon et $\mathrm{al}^{13}$ found that, among traditional risk factors, only hypertension and diabetes were significant independent factors for calcium progression, but findings in previous studies suggested otherwise. ${ }^{14}$ Several studies observed a correlation of most traditional cardiovascular risk factors with increases in existing calcification. $^{12}$

Because the amount of CAC is proportional to disease severity and a graded relationship has been demonstrated between the extent of CAC and the incidence of future cardiovascular events, monitoring CAC over time may be useful in preventing subsequent morbidity and mortality. ${ }^{15}$ In addition to surrogate monitoring of coronary plaque burden over time, a unique potential of serial CAC measurement is the ability to evaluate the success of risk factor modifications and medical treatment. ${ }^{16}$ Electron-beam computed tomography has been shown to accurately assess the clinical efficacy of medical therapies in studies as short as 1 year. ${ }^{17}$ This has the potential to allow primary care providers to tailor treatments for high-risk patients, such as those with known coronary disease, based on disease progression. Current practice calls only for further diagnostic testing, and subsequent invasive interventions-but only once concerning symptoms develop suggesting advanced disease. Here we consider a number of traditional cardiovascular risk factors and demographic factors as each relates to the risk of progressive CAC in a short 2-year interval in subjects without a history of cardiovascular disease (CVD) or renal or liver failure. Future possible implications include tailoring intensive medical management regimens for high-risk patients, stratifying for risk those with known coronary disease, and learning about the limits of using CAC scores.

\section{Methods}

\section{Study Population}

The North Texas Healthy Heart (NTHH) study is a prospective cohort study involving a convenience sample of 571 subjects recruited from 12 sites participating in the North Texas Primary Care Practice-based Research Network (NorTex) from April 2006 to May 2008. NorTex is a collaborative network of primary care clinics serving low-income, underrepresented populations of the Dallas/Fort Worth, Texas, metropolitan area. The 12 family medicine/internal medicine clinic sites that participated in the NTHH study included 4 academic community-based clinics, 3 community health centers, 4 solo-practitioner private practices, and 1 federally qualified health center. Participants were eligible for the study if they were aged $>44$ years; self-identified as non-Hispanic white, non-Hispanic African American, or Hispanic/Latino; and had no history of self-reported CVD (coronary artery disease, peripheral arterial disease, history of myocardial infarction or stroke, or congestive heart failure), renal failure, or liver failure. All participants were consecutively screened for eligibility either on site or via phone from a centralized NorTex research office located within the Department of Family Medicine at the University of North Texas Health Science Center. Initial contact was made with 1062 individuals, of whom 860 met eligibility criteria. Of those who were eligible, 670 individuals were invited and 571 agreed to participate (85\% recruitment rate). A total of 364 participants completed the follow-up annual telephone calls and an in-person study visit at 2 years. The analysis presented here includes the 311 participants who completed multislice computed tomography (MSCT) of the chest at baseline and 2 years. This represents a $58 \%$ follow-up rate over 2 years. All study procedures were approved by the University of North Texas Health Science Center and John Peter Smith Health Network institutional review boards.

\section{Study Procedures}

All participants who consented to participate underwent an initial 1-hour private, in-person interview. Women without a history of hysterectomy underwent a urine pregnancy test; none of these were positive. Participants then completed weight, height, waist-to-hip circumference, and blood pres- 
sure measures at both visits. Height and weight measurements were used to calculate a body mass index (BMI) for each subject using the Quetelet equation (kilograms divided by square meters). ${ }^{18}$ Automated Welch Allyn sphygmomanometers were used to measure heart rate and systolic and diastolic blood pressures in each arm using a appropriately sized cuff. The measures were taken after the participant had been seated quietly for 5 minutes with both feet flat on the floor and the back comfortably supported. Mean heart rate and systolic and diastolic blood pressure were calculated for each subject based on 2 separate measures. These procedures were repeated at the 2-year visit.

\section{Demographic and Health Behavior Measures}

The NTHH study used standardized questions from the Behavioral Risk Factor Surveillance System to collect select demographic and health behavior information. Age (years) was registered as a continuous variable. Race/ethnicity was self-reported and categorized as non-Hispanic white, non-Hispanic African American, Hispanic, and other.

\section{Physiologic and Clinical Measures}

The presence of coronary calcium was measured using 16-slice MSCT, and Agatston units ${ }^{19}$ were calculated. The MSCT took images every $3 \mathrm{~mm}$ from the carina to the base of the heart, and double inspiration was used to minimize motion artifacts. Electrocardiography correlated heart rate with the images produced by Vitrea software (Vital Images (Minnetonka, MN) was used to quantify calcium. Total time in the scanner was 10 to 15 minutes, which was open with no contrast administered. CAC quantification was reviewed and interpreted by a radiologist from Radiology Associates at the Center for Diagnostic Imaging at the University of North Texas Health Science Center; this radiologist was blinded to participant characteristics. These procedures were repeated at the 2-year visit, and the differences from baseline CAC measures were calculated for each participant. Because the resulting values were skewed and transformation attempts failed to normalize the variable, CAC differences were categorized as increase in CAC (CAC difference $>0$ ) or no change or improvement in CAC (CAC difference $\leq 0)$. In addition, each participant was assessed yearly on whether they had suffered a myocardial infarction, stroke, transient ischemic attack, new-onset heart failure, peripheral arterial disease, or any other new heart condition. A composite CVD event was coded as having none or any of these events. CAC and CVD composite outcomes functioned as our dependent variables.

Other clinical factors included hypertension, diabetes, and lipid status. After patients had fasted for 8 hours, blood was collected for serum chemistries and analyzed using a commercial laboratory. Hypertension was considered present if blood pressure was $\geq 140 / 90 \mathrm{mmHg}$, the subject reported being diagnosed with hypertension, or the subject was taking antihypertensive medications. Diabetes was considered present if the fasting glucose concentration was $\geq 126 \mathrm{mg} / \mathrm{dL}$, the subject reported being previously diagnosed with diabetes, or the subject was taking any diabetic medication. Dyslipidemia was considered to be present if, based on Adult Treatment Panel III guidelines, the participant had an $\mathrm{LDL} \geq 160 \mathrm{mg} / \mathrm{dL}$, the participant reported being previously diagnosed with high cholesterol, or the participant was taking a lipid-lowering medication. Because the study was conducted from 2006 to 2008, Adult Treatment Panel III guidelines were used; these define LDL concentrations $\geq 160$ $\mathrm{mg} / \mathrm{dL}$ as high or very high.

Primary independent variables included the presence of hypertension, dyslipidemia, diabetes, or any combination of these chronic conditions and baseline values of LDL, non-HDL, HDL, triglycerides, systolic and diastolic blood pressures, fasting glucose, and BMI. We contemplated assessing the mean of or difference in physiologic measures between clinic visits, but variability at the time of measurement limited the reliability and clinical significance of such measures.

\section{Sample Size and Statistical Analyses}

A retrospective sample size calculation for cohort study designs was conducted based on data from the Multi-Ethnic Study of Atherosclerosis (MESA) published by Budoff et al. ${ }^{20}$ The MESA study found that just more than $50 \%$ of its analytic cohort $(\mathrm{n}=6778)$ had CAC progression with a median follow-up of 7.6 years. Using a relative risk of 1.3 in the likelihood of developing coronary heart disease from progressive CAC changes, as found in the MESA study, we would achieve a desired power of $80 \%$ with a sample size of 167 . These analyses are, in fact, based on a sample of 311 participants, ensuring sufficient power was achieved. 
All statistical analyses were performed using SPSS version 21.0 (IBM, Chicago, IL). Descriptive statistics are provided for all variables. Counts and frequencies are provided for categorical data, and means with standard deviations (SDs) are provided for continuous variables. Independent sample $t$ tests and $\chi^{2}$ analyses were performed to test for differences in independent variables between participants with CAC progression. Differences between CAC progression and a composite CVD event were also assessed. Logistic regression was performed to assess associations between independent variables, CAC progression, and composite CVD events. Unadjusted and adjusted odds ratios and $95 \%$ confidence intervals were calculated. Statistical significance was assessed at the $\alpha=0.05$ level.

The multiple logistic regression model assessed for multicollinearity using tolerance and variation inflation factor, with age and sex variables in the final models. No collinear relationships were identified.

\section{Results}

Table 1 provides demographic information of the population $(\mathrm{N}=311)$ by change in CAC measures. Overall, the average time between baseline MSCT and 2-year follow-up MSCT was 24.44 months (SD, 2.64 months), with a mean change in CAC scores of 24.45 Agatston units (SD, 137.19 Agatston units). CAC did not progress in 218 individuals (70\%), compared with 113 participants (30\%) who demonstrated an increase. Those who had increases in CAC scores were older; were more often male and white; had hypertension, dyslipidemia, or diabetes; and had $\geq 2$ chronic conditions. In addition, those with an increase in CAC scores had unfavorable disease measures with regard to cholesterol, blood pressure, and fasting glucose. No difference was found in BMI measures between the 2 groups. Table 2 demonstrates that among those who experienced a composite CVD event, $69.6 \%$ had an increase in CAC, whereas $30.4 \%$ did not.

Table 3 provides regression results assessing the relationship between an increase in CAC score over 2 years and cardiovascular factors. In crude unadjusted models, every 1-year increase in age was associated with a 1.07 increase in the odds of CAC progressing. Men were $>2$ times more likely than women to have progression. The presence of hy-
Table 1. Population Demographics by Coronary Artery Calcification Change over 2 Years $(\mathrm{N}=311)$

\begin{tabular}{|c|c|c|}
\hline & $\begin{array}{l}\text { Increase in } \\
\text { CAC }\end{array}$ & $\begin{array}{l}\text { No Change or } \\
\text { Improvement in } \\
\text { CAC }\end{array}$ \\
\hline $\begin{array}{l}\text { Time between CAC } \\
\text { measures, months, } \\
\text { mean (SD) }\end{array}$ & $25.56(2.60)$ & $25.31(2.36)$ \\
\hline $\begin{array}{l}\text { Change in CAC, mean } \\
\text { (SD) }\end{array}$ & $82.41(206.78)$ & $-5.59(62.44)$ \\
\hline Age, mean (SD) & $58.40(8.57)$ & $53.95(7.33)$ \\
\hline \multicolumn{3}{|l|}{ Sex } \\
\hline Female & $60(53.1)$ & $152(69.7)$ \\
\hline Male & $53(46.9)$ & $66(30.3)$ \\
\hline \multicolumn{3}{|l|}{ Race/ethnicity } \\
\hline White & $45(39.8)$ & $60(27.5)$ \\
\hline Hispanic/Latino & $33(29.2)$ & $74(33.9)$ \\
\hline African American & $35(31.0)$ & $84(38.5)$ \\
\hline \multicolumn{3}{|l|}{ Disease status at baseline } \\
\hline Hypertension & $66(76.7)$ & $94(61.0)$ \\
\hline Dyslipidemia & $75(69.4)$ & $99(46.3)$ \\
\hline Diabetes mellitus & $25(23.8)$ & $27(12.9)$ \\
\hline \multicolumn{3}{|l|}{ Chronic diseases (n) } \\
\hline 0 & $9(11.0)$ & $41(27.3)$ \\
\hline 1 & $23(28.0)$ & $48(32.0)$ \\
\hline 2 & $34(41.5)$ & $50(33.3)$ \\
\hline 3 & $16(19.5)$ & $11(7.3)$ \\
\hline \multicolumn{3}{|l|}{$\begin{array}{l}\text { Disease measures at } \\
\text { baseline, mean (SD) }\end{array}$} \\
\hline Low-density lipoprotein & $115.66(43.82)$ & $111.10(32.42)$ \\
\hline HDL & $52.24(13.56)$ & $55.62(16.49)$ \\
\hline Triglycerides & $165.52(151.18)$ & $135.97(77.08)$ \\
\hline Non-HDL & $147.12(50.10)$ & $138.28(36.39)$ \\
\hline Systolic blood pressure & $134.95(16.12)$ & $127.59(13.91)$ \\
\hline Diastolic blood pressure & $81.67(9.02)$ & $79.74(8.34)$ \\
\hline Fasting glucose & $107.48(37.20)$ & $97.51(16.92)$ \\
\hline $\begin{array}{l}\text { Body mass index, mean } \\
\text { (SD) }\end{array}$ & $31.21(6.59)$ & $30.42(6.06)$ \\
\hline
\end{tabular}

Data are n (\%) unless otherwise indicated. Values may not add up to 311 because of missing data.

CAC, coronary artery calcification; HDL, high-density lipoprotein; SD, standard deviation.

pertension, dyslipidemia, and diabetes each showed a significant association with CAC progression over 2 years. Moreover, the strength of the association increased with each additional chronic condition; those with all 3 were $>6$ times more likely to have CAC progress in the next 2 years. In terms of serum and physiologic measures, every 1-point increase in triglycerides, systolic blood pressure, and fasting glucose was significantly associated with increased odds of CAC progression. HDL and non-HDL showed a trend toward significance, whereas LDL, diastolic 
Table 2. Composite Cardiovascular Disease Event and Coronary Artery Calcification Change over 2 Years $(\mathrm{N}=311)$

\begin{tabular}{lrr}
\hline \multirow{2}{*}{ CAC Progression } & \multicolumn{2}{c}{ CVD } \\
\cline { 2 - 3 } Increase & Composite Event* & No Event \\
No change/improvement & $7(69.6)$ & $89(31.8)$ \\
$P<.001$ & & $191(68.2)$ \\
\hline
\end{tabular}

Data are n (\%). Values may not add up to 311 because of missing data.

*Experienced a myocardial infarction, stroke, transient ischemic attack, heart failure, peripheral arterial disease, or other heart condition within the previous 2 years.

CAC, coronary artery calcification; CVD, cardiovascular disease.

blood pressure, and BMI showed no association with increase in CAC scores.

After controlling for age and sex in the adjusted regression models, the association of dyslipidemia with CAC progression was maintained, whereas diabetes showed a trend toward significance with CAC progression. Those with dyslipidemia were $>2$ times more likely to have CAC progress. Having $\geq 2$ chronic conditions again showed significant associations and increasing trends with 2-year CAC progression. Those with all 3 chronic conditions were almost 5 times more likely to have CAC progress over 2 years, after adjusting for age and sex. In these adjusted models, the only physiologic factors that remained or became significant were non-HDL, systolic blood pressure, and fasting glucose. That is, for every 1-point increase in nonHDL, systolic blood pressure, and fasting glucose, the odds of CAC progressing showed a statistically significant increase.

Among those with CAC progression, a 4.91 increase was found in the odds of experiencing a composite CVD event (Table 4). This association remained statistically significant after controlling for age, sex, hypertension, dyslipidemia, diabetes mellitus, number of chronic conditions, and BMI.

Table 3. Cardiovascular Factors and Increase in Coronary Artery Calcium Scores over 2 Years

\begin{tabular}{|c|c|c|c|c|c|c|}
\hline & \multicolumn{6}{|c|}{ Change in CAC } \\
\hline & \multicolumn{3}{|c|}{ Unadjusted Model } & \multicolumn{3}{|c|}{ Adjusted Model* } \\
\hline & OR & $95 \% \mathrm{CI}$ & $P$ Value & OR & $95 \% \mathrm{CI}$ & $P$ Value \\
\hline Age & 1.07 & $1.04-1.10$ & $<.001$ & - & - & - \\
\hline \multicolumn{7}{|l|}{ Sex } \\
\hline Female & - & - & - & - & - & - \\
\hline Male & 2.03 & $1.27-3.25$ & .003 & - & - & - \\
\hline \multicolumn{7}{|l|}{ Disease status at baseline } \\
\hline Hypertension & 2.11 & $1.16-3.83$ & .01 & 1.68 & $0.89-3.16$ & .11 \\
\hline Dyslipidemia & 2.64 & $1.62-4.31$ & $<.001$ & 2.19 & $1.32-3.66$ & .003 \\
\hline Diabetes mellitus & 2.11 & $1.15-3.85$ & .02 & 1.91 & $1.01-3.63$ & .05 \\
\hline \multicolumn{7}{|l|}{ Chronic diseases (n) } \\
\hline 0 & - & - & - & - & - & - \\
\hline 1 & 2.18 & $0.91-5.24$ & .08 & 1.71 & $0.69-4.24$ & .25 \\
\hline 2 & 3.10 & $1.33-7.20$ & .01 & 2.47 & $1.02-5.98$ & .04 \\
\hline 3 & 6.63 & $2.31-19.00$ & $<.001$ & 4.80 & $1.57-14.73$ & .01 \\
\hline \multicolumn{7}{|l|}{ Disease measures at baseline } \\
\hline Low-density lipoprotein & 1.00 & $1.00-1.01$ & .29 & 1.01 & $1.00-1.01$ & .10 \\
\hline HDL & 0.99 & $0.97-1.00$ & .06 & 0.99 & $0.97-1.01$ & .18 \\
\hline Triglycerides & 1.00 & $1.00-1.01$ & .04 & 1.00 & $1.00-1.01$ & .08 \\
\hline Non-HDL & 1.01 & $1.00-1.01$ & .07 & 1.01 & $1.00-1.01$ & .04 \\
\hline Systolic blood pressure & 1.03 & $1.01-1.06$ & .002 & 1.03 & $1.01-1.06$ & .01 \\
\hline Diastolic blood pressure & 1.03 & $0.99-1.07$ & .15 & 1.04 & $1.00-1.08$ & .07 \\
\hline Fasting glucose & 1.02 & $1.01-1.03$ & .003 & 1.01 & $1.00-1.02$ & .01 \\
\hline Body mass index & 1.02 & $0.96-1.07$ & .57 & 1.02 & $0.98-1.06$ & .28 \\
\hline
\end{tabular}

*Adjusted for age and sex.

CAC, coronary artery calcification; CI, confidence interval; HDL, high-density lipoprotein; OR, odds ratio. 
Table 4. Coronary Artery Calcium Progression and Development of a Cardiovascular Disease Event over 2 Years

\begin{tabular}{|c|c|c|c|c|c|c|}
\hline \multirow[b]{3}{*}{ CAC Progression } & \multicolumn{6}{|c|}{ Composite CVD Event } \\
\hline & \multicolumn{3}{|c|}{ Unadjusted Model* } & \multicolumn{3}{|c|}{ Adjusted Model ${ }^{\dagger}$} \\
\hline & OR & $95 \% \mathrm{CI}$ & $P$ Value & OR & $95 \% \mathrm{CI}$ & $P$ Value \\
\hline No change/improvement & - & - & - & - & - & - \\
\hline Increase & 4.91 & $1.95-12.35$ & .001 & 4.06 & $1.47-11.21$ & .007 \\
\hline
\end{tabular}

*Experienced a myocardial infarction, stroke, transient ischemic attack, heart failure, peripheral arterial disease, or other heart condition within the previous 2 years.

${ }^{\dagger}$ Adjusted for age, sex, hypertension, dyslipidemia, diabetes mellitus, number of chronic conditions, and body mass index.

CAC, coronary artery calcification; CI, confidence interval; CVD, cardiovascular disease; OR, odds ratio.

\section{Discussion}

In this multiethnic prospective cohort of asymptomatic subjects, age, presence of dyslipidemia, and an increasing number of chronic conditions (hypertension, dyslipidemia, and diabetes) were shown to have a persistent association with 2-year CAC progression after controlling for age and sex. In terms of serum and physiologic disease measures, increases in non-HDL, systolic blood pressure, and fasting glucose were significantly associated with increased odds of CAC progression. Moreover, CAC progression remained independently associated with a composite CVD event after controlling for known risk factors. These findings corroborate several reported associations between traditional cardiovascular risk factors and CAC progression. In addition to data from future studies, these data may signify the value of CAC monitoring as a risk evaluation tool for certain populations, as it was shown to be independently associated with CVD events. The relationships presented also suggest the merit of considering certain risk factors themselves as surrogates for coronary plaque burden over time and avoiding the need for (repeat) computed tomography. $3,15,21-24$

Previous studies of CAC risk factors have focused largely on the incidence rather than the progression of CAC. The studies that do look at progression are scarce and often conflict. Our results on the association of dyslipidemia and an increasing number of chronic conditions support the conclusions of Kronmal et al ${ }^{12}$ and others; this study was also compatible-in terms of the association between lipid measures and systolic blood pressurewith progression of CAC in models adjusted for age, sex, and follow-up time. Consistent with our findings, previous studies with asymptomatic populations reported a positive association of age with
CAC progression. ${ }^{14,25,26}$ Kramer et al ${ }^{27}$ likewise found a positive association of CAC progression with hypertension (odds ratio, 2.11; 95\% confidence interval, 1.33-3.3). Additional studies have supported a relationship between various chronic conditions, including dyslipidemia and hypertension, and CAC progression. ${ }^{13,27-30}$ Studies have also shown an association of blood glucose concentrations and diabetes status with CAC progression. ${ }^{13,27,28,31}$ This effect remained even after adjusting for CAC at baseline. ${ }^{12,32}$

While baseline CAC scores are known to predict future cardiovascular events, ${ }^{3,33,34}$ the importance and clinical value of changes in CAC are less understood. If CAC progression can be established as a reliable surrogate for increasing risk of atherosclerosis and future events, serial assessment of CAC would have many potential clinical applications: to supplement prognostic information for coronary artery disease, to identify subjects who may benefit from more aggressive treatment and/or further diagnostic workup, and to evaluate the efficacy of risk factor modification or other medical treatments on plaque burden. This is particularly true as CAC progression was independently associated with composite CVD events. Our study also identified modifiable risk factors commonly managed in primary and specialty care that are associated with increases in CAC measures, and thereby contributes to ongoing assessment of the clinical importance of CAC progression.

Several limitations of our study must be considered. Wide heterogeneity can be found among existing CAC research study designs and CAC assessment methods. We assumed MSCT is a reliable indicator of CAC, but agreement observed between computed tomography analyses was not considered and, for clinical applicability, the consistency and 
reproducibility of repeat CAC measures should be confirmed. Future studies should exercise diligence in these areas in order to minimize measurement error. ${ }^{11}$ Studies of CAC progression also differ widely on their measurement and classification of calcium change. Progression is typically quantified by Agatston score or calcium volume score and is reported as absolute change, percentage relative change, or change in log calcium plus a constant. Our simple categorization of increase in CAC (CAC difference $>0$ ) versus no change or improvement in CAC (CAC difference $\leq 0)$ makes comparison with other studies questionable. ${ }^{21}$ Indeed, it has been suggested that a large CAC increase is necessary before it can be attributed to pathologic progression. ${ }^{26,35,36} \mathrm{In}$ future, a standardized method of assessing CAC change and a definition of what constitutes meaningful "progression" should be clarified. ${ }^{21}$ The composite CVD measure precludes us from stating the association of CAC progression with any 1 particular CVD outcome.

\section{Conclusion}

As health care providers espouse more evidencebased medicine in their care of patients, more diligence will be applied to the assessment of new innovations in medical care, including the use of cost-benefit analyses and risk-adjusted analyses to estimate clinical outcomes. ${ }^{21} \mathrm{~A}$ small number of individuals with atherosclerosis and detectable coronary calcium will eventually suffer a clinical coronary event, ${ }^{11}$ which was supported by our data. Thus, to be pertinent, serial CAC should be proven as an accurate method for assessing atherosclerotic volume change and have sufficient reproducibility so differences are unlikely to be attributed to measurement error.

A paucity of data exists regarding whether risk factor modification can reduce CAC progression, and guidelines are needed for quantifying subclinical atherosclerosis based on CAC progression. The limited data to date suggest that CAC progression may be a more accurate predictor of future cardiac risk than its baseline measurement, ${ }^{21}$ but there has yet to be a prospective comparison of baseline versus progression of CAC in terms of prognostic value. ${ }^{37}$ While our study only speaks to the association between 2-year CAC changes, traditional risk factors, and composite CVD events, these findings lay the groundwork for future studies to identify those who might benefit from more aggressive treatment and/or further diagnostic workup and to evaluate the efficacy of risk factor modification or other medical treatments on plaque burden. Further studies are needed to determine how the source and extent of traditional cardiovascular risk factors interact with specific levels of CAC progression and the impact this has on cardiovascular morbidity and mortality. In addition, more prospective data are needed to further elucidate the existence and definition of an appropriate population in which CAC monitoring is warranted. ${ }^{38}$

The authors thank the Primary Care Research Center staff of the University of North Texas Health Science Center for providing assistance. The authors also thank the clinicians of the North Texas Primary Care Research Network who helped recruit study participants.

To see this article online, please go to: bttp://jabfm.org/content/ 30/5/592.full.

\section{References}

1. Rumberger JA, Simons DB, Fitzpatrick LA, Sheedy PF, Schwartz RS. Coronary artery calcium area by electron-beam computed tomography and coronary atherosclerotic plaque area. A histopathologic correlative study. Circulation 1995;92:2157-62.

2. Shaw LJ, Raggi P, Schisterman E, Berman DS, Callister TQ. Prognostic value of cardiac risk factors and coronary artery calcium screening for all-cause mortality. Radiology 2003;228:826-33.

3. Arad Y, Goodman KJ, Roth M, Newstein D, Guerci $\mathrm{AD}$. Coronary calcification, coronary disease risk factors, C-reactive protein, and atherosclerotic cardiovascular disease events: the St. Francis Heart Study. J Am Coll Cardiol 2005;46:158-65.

4. Pletcher MJ, Sibley CT, Pignone M, Vittinghoff E, Greenland P. Interpretation of the coronary artery calcium score in combination with conventional cardiovascular risk factors: the Multi-Ethnic Study of Atherosclerosis (MESA). Circulation 2013;128: 1075-64.

5. Baber U, Mehran R, Sartori S, et al. Prevalence, impact, and predictive value of detecting subclinical coronary and carotid atherosclerosis in asymptomatic adults: the BioImage study. J Am Coll Cardiol 2015;65:1065-74.

6. Goff DC Jr, Lloyd-Jones DM, Bennett G, et al. 2013 ACC/AHA guideline on the assessment of cardiovascular risk: a report of the American College of Cardiology/American Heart Association Task Force on Practice Guidelines. J Am Coll Cardiol 2014;63(25 Pt B):2935-59. 
7. Arad Y, Spadaro LA, Goodman K, Newstein D, Guerci AD. Prediction of coronary events with electron beam computed tomography. J Am Coll Cardiol 2000;36:1253-60.

8. Budoff MJ, Gul KM. Expert review on coronary calcium. Vasc Health Risk Manag 2008;4:315-24.

9. Budoff MJ, Achenbach S, Blumenthal RS, et al. Assessment of coronary artery disease by cardiac computed tomography. Circulation 2006;114:1761-91.

10. Blaha MJ, Silverman MG, Budoff MJ. Is there a role for coronary artery calcium scoring for management of asymptomatic patients at risk for coronary artery disease? Clinical risk scores are not sufficient to define primary prevention treatment strategies among asymptomatic patients. Circ Cardiovasc Imaging 2014;7:398-408.

11. Wexler L, Brundage B, Crouse J, et al. Coronary artery calcification: pathophysiology, epidemiology, imaging methods, and clinical implications A statement for health professionals from the American Heart Association. Circulation 1996;94:1175-92.

12. Kronmal RA, McClelland RL, Detrano R, et al. Risk factors for the progression of coronary artery calcification in asymptomatic subjects results from the Multi-Ethnic Study of Atherosclerosis (MESA). Circulation 2007;115:2722-30.

13. Yoon H-C, Emerick AM, Hill JA, Gjertson DW, Goldin JG. Calcium Begets Calcium: Progression of Coronary Artery Calcification in Asymptomatic Subjects. Radiology 2002;224:236-41.

14. Janowitz WR, Agatston AS, Kaplan G, Viamonte M. Differences in prevalence and extent of coronary artery calcium detected by ultrafast computed tomography in asymptomatic men and women. Am J Cardiol 1993;72:247-54.

15. Wong ND, Hsu JC, Detrano RC, Diamond G, Eisenberg H, Gardin JM. Coronary artery calcium evaluation by electron beam computed tomography and its relation to new cardiovascular events. Am J Cardiol 2000;86:495-8.

16. Koulaouzidis G, Charisopoulou D, Maffrett S, Tighe M, Jenkins P, McArthur T. Coronary artery calcification progression in asymptomatic individuals with initial score of zero. Angiology 2013;64:494-7.

17. Budoff MJ, Raggi P. Coronary artery disease progression assessed by electron-beam computed tomography. Am J Cardiol 2001;88:46-50.

18. Keys A, Fidanza F, Karvonen MJ, Kimura N, Taylor HL. Indices of relative weight and obesity. J Chronic Dis 1972;25:329-43.

19. Agatston AS, Janowitz WR, Hildner FJ, Zusmer NR, Viamonte M, Detrano R. Quantification of coronary artery calcium using ultrafast computed tomography. J Am Coll Cardiol 1990;15:827-32.

20. Budoff MJ, Young R, Lopez VA, et al. Progression of coronary calcium and incident coronary heart disease events: MESA (Multi-Ethnic Study of Atherosclerosis). J Am Coll Cardiol 2013;61:1231-9.
21. McEvoy JW, Blaha MJ, DeFilippis AP, et al. Coronary artery calcium progression: an important clinical measurement? A review of published reports. J Am Coll Cardiol 2010;56:1613-22.

22. Kondos GT, Hoff JA, Sevrukov A, et al. Electronbeam tomography coronary artery calcium and cardiac events a 37-month follow-up of 5635 initially asymptomatic low-to intermediate-risk adults. Circulation 2003;107:2571-6.

23. Becker A, Leber A, Becker C, Knez A. Predictive value of coronary calcifications for future cardiac events in asymptomatic individuals. Am Heart J 2008;155:154-60.

24. Callister TQ, Cooil B, Raya SP, Lippolis NJ, Russo DJ, Raggi P. Coronary artery disease: improved reproducibility of calcium scoring with an electronbeam CT volumetric method. Radiology 1998;208: 807-14.

25. Wong ND, Kouwabunpat D, Vo AN, et al. Coronary calcium and atherosclerosis by ultrafast computed tomography in asymptomatic men and women: relation to age and risk factors. Am Heart J 1994;127:422-30.

26. Devries S, Wolfkiel C, Fusman B, et al. Influence of age and gender on the presence of coronary calcium detected by ultrafast computed tomography. J Am Coll Cardiol 1995;25:76-82.

27. Kramer CK, Von Mühlen D, Gross JL, Laughlin GA, Barrett-Connor E. Blood pressure and fasting plasma glucose rather than metabolic syndrome predict coronary artery calcium progression: the Rancho Bernardo Study. Diabetes Care 2009;32:141-6.

28. Lee KK, Fortmann SP, Fair JM, et al. Insulin resistance independently predicts the progression of coronary artery calcification. Am Heart J 2009;157: $939-45$

29. Taylor AJ, Bindeman J, Le TP, et al. Progression of calcified coronary atherosclerosis: relationship to coronary risk factors and carotid intima-media thickness. Atherosclerosis 2008;197:339-45.

30. Tamashiro M, Iseki K, Sunagawa O, et al. Significant association between the progression of coronary artery calcification and dyslipidemia in patients on chronic hemodialysis. Am J Kidney Dis 2001;38: 64-9.

31. Maahs DM, Ogden LG, Kinney GL, et al. Low plasma adiponectin levels predict progression of coronary artery calcification. Circulation 2005;111:747-53.

32. Snell-Bergeon JK, Hokanson JE, Jensen L, et al. Progression of coronary artery calcification in type 1 diabetes: the importance of glycemic control. Diabetes Care 2003;26:2923-8.

33. Raggi P, Callister TQ, Shaw LJ. Progression of coronary artery calcium and risk of first myocardial infarction in patients receiving cholesterol-lowering therapy. Arterioscler Thromb Vasc Biol 2004;24: 1272-7. 
34. Taylor AJ, Bindeman J, Feuerstein I, Cao F, Brazaitis M, O'Malley PG. Coronary calcium independently predicts incident premature coronary heart disease over measured cardiovascular risk factors: mean three-year outcomes in the Prospective Army Coronary Calcium (PACC) project. J Am Coll Cardiol 2005;46:807-14.

35. Detrano R, Wang S, Tang W, et al. Progression of coronary calcification can be tracked with ultrafast computed tomography: concordance and reliability are sufficient. Am J Card Imaging 1994;8(Suppl 1):6.

36. Detrano R, Wang S, Tang W, Brundage B, Wong N. Thick slice electron-beam tomographic scanning allows reproducible and accurate assessments of coronary calcific deposits. Circulation 1995;92(Suppl 1): 1-L50.

37. Azen SP, Mack WJ, Cashin-Hemphill L, et al. Progression of coronary artery disease predicts clinical coronary events. Long-term follow-up from the Cholesterol Lowering Atherosclerosis Study. Circulation 1996;93:34-41.

38. Pandey AK, Blaha MJ, Sharma K, et al. Family history of coronary heart disease and the incidence and progression of coronary artery calcification: Multi-Ethnic Study of Atherosclerosis (MESA). Atherosclerosis 2014;232:369-76. 